\title{
Autism-specific maternal autoantibodies recognize critical proteins in developing brain
}

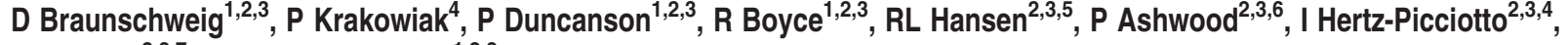 \\ IN Pessah ${ }^{2,3,7}$ and J Van de Water ${ }^{1,2,3}$
}

\begin{abstract}
Autism spectrum disorders (ASDs) are neurodevelopmental in origin, affecting an estimated 1 in 88 children in the United States. We previously described ASD-specific maternal autoantibodies that recognize fetal brain antigens. Herein, we demonstrate that lactate dehydrogenase A and B (LDH), cypin, stress-induced phosphoprotein 1 (STIP1), collapsin response mediator proteins 1 and 2 (CRMP1, CRMP2) and Y-box-binding protein to comprise the seven primary antigens of maternal autoantibody-related (MAR) autism. Exclusive reactivity to specific antigen combinations was noted in $23 \%$ of mothers of ASD children and only $1 \%$ of controls. ASD children from mothers with specific reactivity to LDH, STIP1 and CRMP1 and/or cypin (7\% vs $0 \%$ in controls; $P<0.0002$; odds ratios of 24.2 (95\% confidence interval: 1.45-405)) had elevated stereotypical behaviors compared with ASD children from mothers lacking these antibodies. We describe the first panel of clinically significant biomarkers with over $99 \%$ specificity for autism risk thereby advancing our understanding of the etiologic mechanisms and therapeutic possibilities for MAR autism.
\end{abstract}

Translational Psychiatry (2013) 3, e277; doi:10.1038/tp.2013.50; published online 9 July 2013

\section{Introduction}

Autism spectrum disorders (ASDs) are a group of etiologically and phenotypically heterogeneous neurodevelopmental disorders manifesting in early childhood, currently estimated at a prevalence of 1 in 88 children. ${ }^{1}$ ASD is defined by core deficits in communication and reciprocal social interaction, and by the presence of repetitive or stereotypical behaviors. ${ }^{2}$ Findings of dysregulated immune function, ${ }^{3,4}$ neuroinflammation, ${ }^{5}$ as well as the presence of maternal autoantibodies directed against rodent, ${ }^{6}$ human ${ }^{7}$ and non-human primate ${ }^{8}$ fetal brain tissue, strongly support an etiological role for the immune system in some forms of ASD. We first observed immunoreactivity to proteins at approximately 37 and $73 \mathrm{kDa}$ exclusively in the mothers of children with an $A S D,{ }^{7}$ and subsequently confirmed these findings in an expanded cohort, while demonstrating that maternal reactivity to the 37 and $73 \mathrm{kDa}$ antigens were associated with increased severity of language deficits in the offspring. ${ }^{8}$ Furthermore, we noted reactivity to an additional pair of bands in the region of 39 and $73 \mathrm{kDa}$ that associated significantly with increased irritability and self-injurious behavior in the children of positive mothers. We have also demonstrated that the functional MET promoter variant rs $1858830 \mathrm{C}$ allele is associated with the presence of these specific maternal autoantibodies. ${ }^{9}$

An etiological role for maternal antibodies in ASD is plausible because of the gestational transfer of maternal IgG during pregnancy where maternal $\lg G$ is detected in fetal circulation as early as 13 weeks of gestation in humans. By 30 weeks of gestation, levels in the fetal compartment reach approximately $50 \%$ of circulating levels in the mother, ${ }^{10}$ with levels at birth exceeding maternal IgG levels. ${ }^{11}$ The developing blood-brain barrier is actively changing during fetal neurodevelopment and is permissive to IgG molecules during this period. ${ }^{12}$ Moreover, studies in rodents ${ }^{13-15}$ and nonhuman primates ${ }^{16}$ have identified ASD-like behavioral impairments in offspring born to dams exposed during pregnancy to passively transferred human IgG from mothers with brainreactive antibodies that were not observed in animals exposed to control IgG.

Recognizing that identification of the target antigens for MAR autism is the next critical step toward advancing this area of research, we employed a proteomic approach to attain this goal. The identity of each of the candidate antigens was successfully determined by tandem mass spectrometry peptide sequencing, and subsequently confirmed with western blotting experiments using purified target proteins. Further, we characterized behavioral outcomes in the children of MAR-positive mothers that associated with the presence of the most common MAR pattern.

\section{Materials and methods}

Study subjects. Consenting mothers were enrolled through the Center for Children's Environmental Health as part of the

\footnotetext{
${ }^{1}$ Department of Internal Medicine, University of California at Davis, Davis, CA, USA; ${ }^{2}$ Davis M.I.N.D. Institute, University of California, Davis, CA, USA; ${ }^{3}$ Children's Center for Environmental Health, University of California at Davis, Davis, CA, USA; ${ }^{4}$ Division of Epidemiology, Department of Public Health Sciences, University of California at Davis, Davis, CA, USA; ${ }^{5}$ Department of Pediatrics, University of California at Davis, Davis, CA, USA; ${ }^{6}$ Department of Medical Microbiology, University of California at Davis, Davis, CA, USA and ${ }^{7}$ Department of Molecular Biosciences, School of Veterinary Medicine, University of California at Davis, Davis, CA, USA

Correspondence: Professsor J Van de Water, Division of Rheumatology, Allergy and Clinical Immunology, Department of Internal Medicine, University of California at Davis, 451 E Health Sciences Drive, Suite 6510; Davis, CA 95616, USA.

Email: javandewater@ucdavis.edu

Keywords: autism; autoantibodies; fetal brain; neurodevelopment

Received 2 January 2013; revised 22 April 2013; accepted 23 April 2013
} 
continuing CHARGE (CHildhood Autism Risks from Genetics and Environment) Study at the M.I.N.D. Institute at the University of California at Davis as described previously. ${ }^{17}$ This study protocol followed the ethical guidelines of the most recent Declaration of Helsinki, and was approved by the institutional review boards at the University of California, Davis, the State of California Department of Developmental Services and the University of Southern California. Informed consent was obtained before participation.

Recruitment, eligibility and psychometric assessment protocols have been previously described..$^{17,18}$ The CHARGE study participants in this study included children diagnosed with autism or ASD $(n=246)$, and children selected from the general population (typically developing (TD); $n=149$ ). Diagnosis of all enrolled children was confirmed at the UC Davis M.I.N.D. Institute. The diagnosis of ASD was based on the Autism Diagnostic Observation Schedule ${ }^{19}$ and the Autism Diagnostic Interview-Revised. ${ }^{20}$ All other children were screened on the Social Communication Questionnaire, and those scoring at or above the cutoff were then assessed using the ADI-R and Autism Diagnostic Observation Schedule. A diagnosis of typical development was assigned to general population controls based on the Social Communication Questionnaire and composite scores of 70 or higher for the Mullen Scales of Early Learning ${ }^{21}$ and the Vineland Adaptive Behavioral Scales. ${ }^{22}$ In addition, all mothers of children enrolled in this study completed the Aberrant Behavior Checklist $(\mathrm{ABC}),{ }^{23}$ which consists of 58 questions designed to measure the severity of several deviant behaviors, yielding subscores in the domains of irritability, lethargy, stereotypy, hyperactivity and inappropriate speech. Higher scores indicate more severe aberration.

Sample collection. Maternal blood was collected in acid citrate dextrose tubes (BD Diagnostic, Franklin Lakes, NJ, USA). Plasma was separated from cells, coded and aliquoted to minimize freeze/thaw cycles then stored at $-80^{\circ} \mathrm{C}$ until use.
Fetal brain antigen preparation. Banked fetal Rhesus macaque brain (FRB) of 152 days gestation from the California National Primate Research Center and used to prepare a protein extract as described previously. ${ }^{8}$ Briefly, tissue was homogenized in a detergent buffer containing a phosphatase and protease inhibitor cocktail (Roche Complete, Roche, Mannheim, Germany) and sonicated. Insoluble material was removed by centrifugation and a buffer exchange performed against $50 \mathrm{~mm}$ Tris- $\mathrm{HCl}$ containing $1 \%$ lithium dodecyl sulfate. Protein concentration was determined using the bicinchoninic acid reaction (Pierce, Rockford, IL, USA) and adjusted to $4.5 \mathrm{mg} \mathrm{ml}^{-1}$.

Western blot. For initial screening of maternal plasma samples, $300 \mu \mathrm{g}$ of prepared FRB was separated under reducing conditions in a prep well $4-12 \%$ gradient SDSPAGE mini-gel (Invitrogen, Carlsbad, CA, USA) and transferred to nitrocellulose. The nitrocellulose strips were probed with maternal plasma diluted 1:400, washed and incubated with 1:20000 diluted horseradish peroxidase conjugated goat anti-human IgG (Invitrogen). The strips were then washed, incubated with SuperSignal West Chemilluminescent Substrate (Pierce), and imaged using a FluorChem 8900 imager using AlphaEaseFC software (Protein Simple, Santa Clara, CA, USA). Mothers found to react against FRB were subsequently used for proteomic antigen identification.

Prep cell fractionation. To enrich for target proteins at the observed molecular weights, and to provide material for subsequent two-dimensional (2D) gel analysis, $100 \mathrm{mg}$ of FRB was first fractionated using a Prep Cell apparatus (Bio-Rad, Hercules, CA, USA). Briefly, FRB was electrophoresed through a $28-\mathrm{mm}$ cylindrical $10 \%$ poly-acrylamide gel for $17 \mathrm{~h}$ at $12 \mathrm{~W}$. A total of 110 fractions were collected at $5 \mathrm{~min}$ intervals at a flow rate of $0.75 \mathrm{ml} \mathrm{min}^{-1}$. Fractions were concentrated to $5 \mathrm{mg} \mathrm{ml}^{-1}$ using Amicon Ultra-4 with Ultracel-10k membranes (Millipore, Cork, Ireland) and assayed by western blot to determine molecular weight and
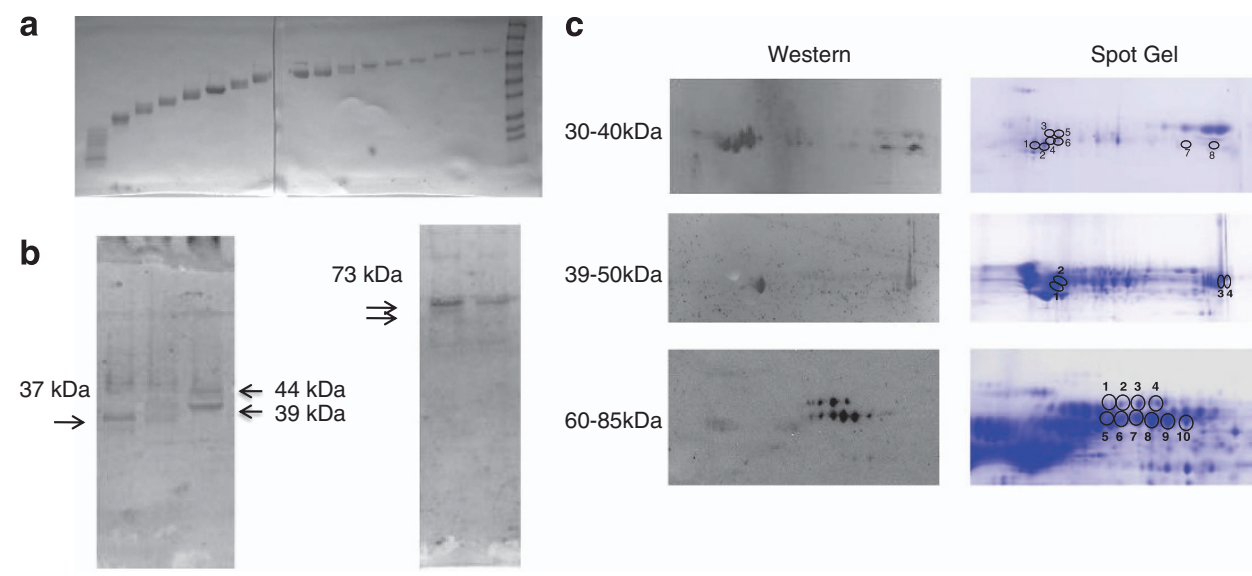

Figure 1 Prep cell protein fractionation and two-dimensional (2D) gel. (a) Ponceau stained nitrocellulose membrane containing samples from every sixth fraction collected from Prep Cell separation of Rhesus fetal brain protein. (b) Western blots of the membranes in a probed with maternal plasma reactive against the target bands. (c) Duplicate 2D gels were run with target region 30-40 kDa, 39-50 kDa and 60-85 kDa optimized Prep Cell fractions. The left column shows chemiluminescent images of 2D western blot probed with diluted plasma from mothers reactive against each of the antigens. Circles on the gels in the right column represent spots of reactivity between maternal antibodies and cognate antigens on the $2 \mathrm{D}$ blots on the left that were used to guide spot picking. 
verify antigen reactivity (Figure 1). Ponceau staining of western blot membranes confirmed substantial enrichment for proteins by molecular weight, yielding fractions with a range of approximately $5-10 \mathrm{kDa}$, which facilitated subsequent analysis. Fractions containing proteins in the targeted molecular weight ranges were selected for identification by 2D gel electrophoresis.

2D Electrophoresis. Protein fractions derived from Prep Cell separation that were enriched for antigens recognized by maternal antibodies were separated by $2 \mathrm{D}$ electrophoresis. Thirty microgram each of targeted fractions were labeled with Cy2 (GE Life Sciences, Pittsburgh, PA, USA) and prepared for 2D gel electrophoresis, and all 2D gels were performed in duplicate. Fifteen microgram of each sample was loaded into each of two $\mathrm{pH} 3-10$ isoelectric focusing strips (GE Healthcare, Piscataway, NJ, USA) and separated to equilibrium. The strips were then rinsed and loaded onto $10.5 \%$ (lower molecular weight) or $8.5 \%$ (higher molecular weight) polyacrylamide gels (GE Healthcare) for second dimension electrophoresis. After electrophoresis, fluorescence images of the gels were acquired and verified for consistency using Image Quant software (version 6.0, GE Healthcare). One of the duplicate gels was transferred electrophoretically to a $0.2-\mu \mathrm{m}$ pore nitrocellulose membrane (GE Healthcare) and a western blot performed using maternal plasma samples reactive to the band region of interest. The blot image size was adjusted using internal markers to match identically with the non-transferred $2 \mathrm{D}$ gel for spot picking alignment. The spots identified on the western blot image were picked from the 2D gel using an Ettan Spot Picker (GE Healthcare).

Mass spectrometry. Spots identified by western blot were mapped back to and picked from the duplicate 2D gel, were subsequently washed and digested with trypsin (Promega, Madison, WI, USA). The tryptic peptides were desalted using a Zip-tip C18 (Millipore, Billerica, MA, USA) and spotted on the matrix-assisted laser desorption/ionization plate (model ABI 01-192-6-AB). Matrix-assisted laser desorption/ionization time of flight mass spectrometry and time of flight/time of flight tandem MS/MS spectra were obtained in a data-dependent acquisition mode on an $A B I$ 4700 mass spectrometer (Applied Biosystems, Framingham, MA, USA). Matrix-assisted laser desorption/ionization time of flight mass spectra and time of flight/time of flight tandem MS fragmentation spectra averaged 4000 laser shots per fragmentation spectrum on each of the 10 most abundant ions present in each sample. Mass accuracy in MS/MS mode was better than $\pm 100 \mathrm{mDa}$. Approximately 22 peptides were sequenced for each protein hit resulting in minimum sequence coverage of $46 \%$. Both the resulting peptide mass and the associated fragmentation spectra were analyzed by a GPS Explorer workstation equipped with MASCOT search engine (Matrix Science, Boston, MA, USA) and used to query the National Center for Biotechnology Information non-redundant database. Searches were performed without constraining protein molecular weight or isoelectric point, with variable carbamidomethylation of cysteine and oxidation of methionine residues, and with one missed cleavage also allowed in the search parameters. Candidates with either protein score confidence interval $(\mathrm{Cl} \%)$ or ion $\mathrm{Cl} \%$ of greater than $95 \%$ were considered positive.

Antigen verification and autoantibody specificity western blots. After proteomic antigen identification, pure candidate proteins were analyzed by western blot strip with gel loading of $4 \mu \mathrm{g}$ for each of the candidates. Tested proteins included purified native lactate dehydrogenase (LDH; Cell Sciences, Canton, MA, USA) containing the A and $B$ subunits, recombinant full-length guanine deaminase (cypin), stress-induced phosphoprotein 1 (STIP1; Abnova, Taipei, Taiwan) and recombinant full-length collapsin response mediator proteins 1 and 2 (CRMP1, CRMP2) and Y-Box-binding protein 1 (YBX1; OriGene, Rockville, MD, USA). Recombinant proteins produced in-house were also used in these western blots and their production is described below. Commercially available polyclonal antibodies for LDH, cypin, CRMP1 and CRMP2, YBX1 and STIP1 (Abcam, Cambridge, MA, USA) diluted at 1:20000 were used as positive controls and species-specific, HRP conjugated secondary antibodies were used at a 1:20000 dilution for detection.

Cell culture. Recombinant proteins were produced using transient transfection of Human Embryonic Kidney cells with pCMV6-Entry vector (Origene) containing full-length sequences of LDH, cypin, CRMP1, YBX1 or STIP1 with a DDK tag according to the manufacturer's protocol. Briefly, at $70 \%$ confluence, the Human Embryonic Kidney cells were transfected with $10 \mu \mathrm{g}$ plasmid DNA with $30 \mu \mathrm{l}$ of MegaTran 1.0 transfection reagent (Origene) for $24 \mathrm{~h}$. After $24 \mathrm{~h}$, cells were harvested, lysed, and protein was prepared as described above for Rhesus macaque fetal (GD 152) brain (RFB) samples. Recombinant proteins were then purified form the crude mixture using anti-FLAG M2 affinity gel (Sigma-Aldrich, St Louis, MO, USA). Protein concentration was determined and purity was verified by western blot using commercial antibodies.

Blocking studies. To verify antigen-binding specificity, diluted (1:400) maternal plasma samples were incubated with $400 \mu \mathrm{g}$ of purified recombinant LDH, cypin, YBX1, CRMP1, CRMP2 or STIP1, or with buffer alone for $20 \mathrm{~h}$ at $4{ }^{\circ} \mathrm{C}$. The absorbed plasma samples were then used to probe western blot strips containing RFB protein and imaged as described above.

Statistical analysis. Antigen reactivity prevalence data were analyzed using a two-way group comparison of ASD vs TD with individual antigens, and groups of antigens using a Fisher's exact test. Differences were considered significant at $P<0.05$, and odds ratios (ORs) with $95 \% \mathrm{Cls}$ were calculated for all significant associations. Among children with ASD, combined behavioral scores were compared based on maternal band reactivity using unpaired two-tailed $t$-tests, after confirming equal variances. A 0.5 continuity correction was applied to all odds ratio calculations for observations with zero cell counts. ${ }^{24}$ Small sample size adjustment of OR and $95 \%$ confidence limits ${ }^{25}$ was 
implemented using $\mathrm{R}$ code (http://rss.acs.unt.edu/Rdoc/ library/epitools/html/oddsratio.html). Behavioral associations with autoantibody pattern were analyzed by analysis of covariance adjusted for child's age; very little difference in coefficients or standard errors was noted when age was taken into account. All statistical analysis was carried out using SAS software (SAS Institute, Cary, NC, USA).

\section{Results}

Antigen identification. To provide an enriched fraction of RFB homogenate for mass spectrometric analysis, the RFB homogenate was separated into 110 fractions by molecular mass using a size fractionation column. Fractions containing proteins in the target regions of $30-40 \mathrm{kDa}, 39-50 \mathrm{kDa}$ and 60-85 kDa were each analyzed individually on separate pairs of 2D gels for a total of six gels (Figure 1a). One 2-D gel from each pair was transferred to nitrocellulose and probed with diluted maternal plasma from a mother of a child with ASD who displayed reactivity to the bands in the region of interest. Positive spots were observed on each of the 2D western blots, and all identified spots were selected for mass spectrometric analysis. The protein with the highest confidence (98-100\%) from each spot was selected for additional verification by western blot (Figure 1b).

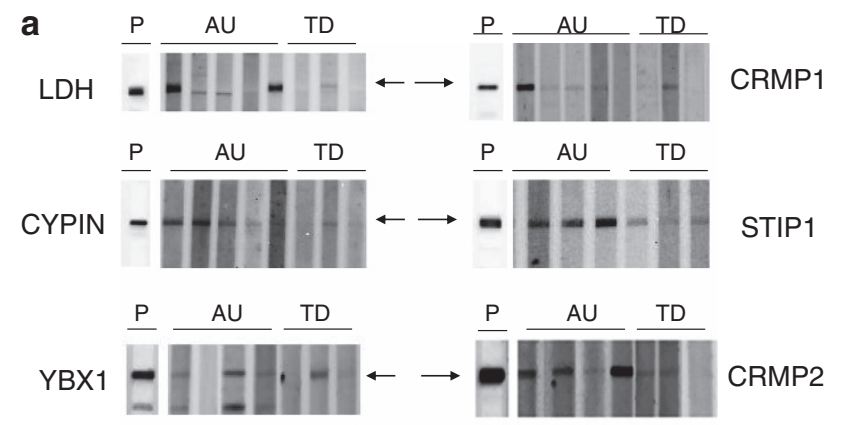

b

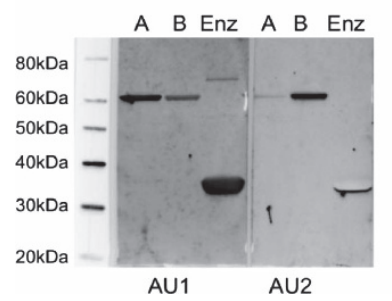

Figure 2 Western blots of candidate antigens. (a) Purified lactate dehydrogenase ( $\mathrm{LDH}$, containing $A$ and $B$ subunits) or recombinant full-length Cypin, $Y$-boxbinding protein (YBX1), collapsin response mediator protein (CRMP) 1, CRMP2 and stress-induced phosphoprotein 1 (STIP1) proteins were probed with plasma from mothers of children with autism (AU) or mothers of typically developing (TD) controls diluted to 1:400, $\mathrm{P}$ indicates polyclonal antibody positive controls. Arrows indicate location of band(s) of interest. Note the different patterns of reactivity to LDH A and $B$ subunits in lanes $1-3$ of the LDH panel. This reactivity is further characterized in panel $\mathbf{b}$ where the blot of recombinant human glutathion-S-transferase (GST)tagged LDHA (A), recombinant human GST-tagged LDHB (B) or purified native human $\mathrm{LDH}$ (Enz) was probed with maternal plasma diluted to 1:400, demonstrating variable reactivity to the LDH subunits. Also of note, as demonstrated in Table 1, some mothers of typically developing children have antibodies to the individual proteins and it is reactivity to the specific antigen combinations that confers specificity for MAR autoantibodies.
Antigen verification. Commercially available purified or full-length recombinant human proteins were used to verify maternal antibody reactivity to the identified antigens by western blot analysis (Figure 2a). Among the antigens tested in an initial screen of our candidate proteins, maternal IgG reactivity to $\mathrm{LDH}$ ( $37 \mathrm{kDa}$ band), YBX1 (39 kDa band), STIP1 (upper $73 \mathrm{kDa}$ band) and CRMP1 (lower $70 \mathrm{kDa}$ band) or cypin, (a 44-kDa protein not noted in our initial studies) was observed in more than $75 \%$ of mothers of children with ASD $(n=50)$ tested for reactivity, based upon their original fetal monkey brain autoantibody profile. These candidate proteins were thus used for screening of the larger cohort. Detection of subunit specificity for LDH-reactive maternal antibodies was carried out using purified human LDH and full-length recombinant human LDHA and LDHB subunits by western blot (Figure 2b). The LDHA and LDHB subunits share approximately $90 \%$ sequence homology and although some mothers had reactivity individually to LDHA or LDHB, most maternal antibodies that bound to $\mathrm{LDH}$ recognized both subunits. Screening of all maternal plasma samples was thus carried out with purified LDH containing both subunits.

Binding inhibition of maternal antibodies. To further verify that the candidate autoantigens corresponded to the targeted fetal brain protein bands, we performed binding inhibition studies. For the target antigen corresponding to the $37 \mathrm{kDa}$ band, diluted maternal plasma samples were incubated overnight with or without purified human LDH, and then used to probe western blots containing RFB protein (Figure 3). Preincubation with LDH abolished any reactivity to fetal brain protein in maternal plasma samples previously shown to be positive for the $37-\mathrm{kDa}$ band, while reactivity to other bands was unaffected. Blocking studies involving YBX1, cypin, CRMP1, CRMP2 and STIP1 yielded similar

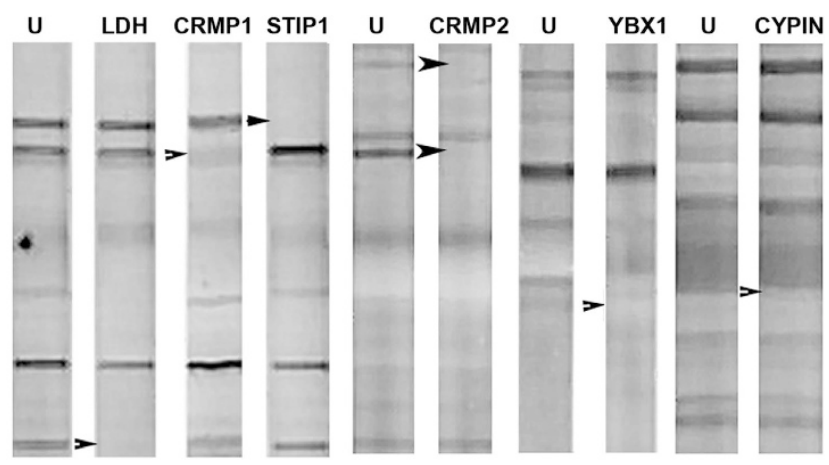

Figure 3 Blocking western blot. Maternal plasma samples incubated without any target antigen (U-unblocked) or blocked with lactate dehydrogenase (LDH), collapsin response mediator protein (CRMP) 1, stress-induced phosphoprotein 1 (STIP1; mother with reactivity to all three proteins), CRMP2 (mother who is CRMP1 - and CRMP2 +), Y-box-binding protein (YBX1) and Cypin (mother with reactivity to Cypin, STIP1 and CRMP1) were used to probe a western blot containing Rhesus macaque fetal brain. The pure recombinant proteins were incubated with the plasma sample diluted 1:400 overnight, centrifuged and applied to the membrane blot containing fetal monkey brain. Arrowheads indicate location of the target antigen removed by overnight pre-incubation. Note that only the specific band(s) is/are removed following pre-incubation with each individual target protein indicating no cross-reactivity between the candidate antigens. 
results (Figure 3). Thus, it was verified that the bands targeted by our proteomic search were accurately identified.

Autoantibody specificity. To determine autoantibody prevalence, we assessed 246 mothers of children diagnosed with ASD and 149 mothers of typically developing children from the CHARGE (Childhood Autism Risks from Genetics and Environment) Study for reactivity to the individual candidate antigens by western blot. Maternal reactivity to any of the antigens, individually or in combination, was highly significantly associated with an outcome of ASD in the child (Table 1) with an odds ratio of 3.26 (95\% Cl: 1.92-5.53). We further found that, as in our original discovery, western blot analysis of fetal brain proteins reactivity to $\mathrm{LDH}(37 \mathrm{kDa}$ band) in any context was significantly more frequent for the mothers of children with ASD $(P=0.0012)$ compared with the mothers of the TD controls (Table 1). Similarly, the presence of autoantibodies to CRMP1, CRMP2 or STIP1 was also significantly associated with ASD $(P=0.0034, P=0.004$ and
$P<0.0001$, respectively; Table 1). In addition, there were several combinations of autoantibodies that were highly specific to ASD and were not found in the plasma of TD mothers. The most specific patterns of reactivity were to the LDH/STIP1/CRMP1 (5\% ASD vs 0\% TD; corresponds to the original $37 / 73 \mathrm{kDa}$ band pattern) and LDH/STIP1/CRMP1/ cypin ( $2 \%$ vs $0 \%$ TD) combinations $(P=0.0025$, OR: 8.3 (95\% Cl: 1-293) and OR: 3.1 (95\% Cl: 0.37-124); Table 1). Also notable was the cypin/STIP1/CRMP1 pattern found in 18 mothers whose children were diagnosed with ASD and only one mother of a child enrolled as typically developing $(P=0.0025$; Table 1). There were several additional patterns that were found only in the mothers of ASD children as noted in Table 1. Furthermore, numerous autoantibody combinations were also significantly associated with, but not specific to, ASD (Table 1). When combined, a total of nearly $23 \%$ of mothers of ASD children had a combination of autoantibody patterns containing two or more of the target proteins ( $P<0.0001$, OR: 21.7 (95\% Cl: 5.2-90; Table 1).

Table 1 Summary of maternal antibody reactivity to the target autoantigens

\begin{tabular}{|c|c|c|c|c|}
\hline Antigen/antigen combinations & $A U / A S D, \%(\mathrm{n}=246)$ & $T D, \%(\mathrm{n}=149)$ & $\mathrm{P}-$ value $^{\mathrm{a}}$ & OR $(95 \% \mathrm{Cl})$ \\
\hline Reactivity to any antigen ${ }^{\mathrm{b}}$ & 89 (218) & 70 (105) & $<0.0001$ & $3.26(1.92-5.53)$ \\
\hline \multicolumn{5}{|l|}{ Individual proteins } \\
\hline LDH & $28(68)$ & $13(20)$ & 0.0012 & $2.5(1.4-4.3)$ \\
\hline Cypin & $25(62)$ & $19(29)$ & 0.22 & $1.4(0.8-2.3)$ \\
\hline STIP1 & 59 (145) & 36 (53) & $<0.0001$ & $2.6(1.7-4)$ \\
\hline CRMP1 & $32(78)$ & $18(27)$ & 0.0034 & $2.1(1.3-3.4)$ \\
\hline CRMP2 & $18(44)$ & $7(11)$ & 0.004 & $2.7(1.4-5.5)$ \\
\hline YBX1 & $31(78)$ & $23(34)$ & 0.065 & $1.57(0.98-2.5)$ \\
\hline \multicolumn{5}{|l|}{ Significant combinations } \\
\hline LDH + STIP1 & $16(40)$ & $6(9)$ & $0.0026^{c}$ & $3.0(1.4-6.4)$ \\
\hline LDH + CRMP1 & $9(22)$ & $3(4)$ & $0.0196^{c}$ & $3.6(1.2-10.5)$ \\
\hline Cypin + STIP1 & $18(45)$ & $8(12)$ & $0.0049^{\mathrm{c}}$ & $2.6(1.3-5)$ \\
\hline Cypin + CRMP1 & $9(22)$ & $3(5)$ & $0.038^{\mathrm{c}}$ & $2.8(1-7.6)$ \\
\hline STIP1 + CRMP1 & $18(45)$ & $7(10)$ & $0.0014^{c}$ & $3.1(1.5-6.4)$ \\
\hline \multicolumn{5}{|l|}{ Specific combinations } \\
\hline \multicolumn{5}{|l|}{ Combinations of two antigens } \\
\hline LDH + YBX 1 & $2(4)$ & $0(0)$ & $0.16^{\mathrm{c}}$ & $3.1(0.37-124)^{d}$ \\
\hline LDH + CRMP2 & $1(2)$ & $0(0)$ & $0.5^{\mathrm{c}}$ & $2.18(0.15-67)^{d}$ \\
\hline YBX1 + CRMP2 & $5(12)$ & $0.6(1)$ & $0.037^{\mathrm{C}}$ & $7.6(0.97-59)^{d}$ \\
\hline CRMP1 + CRMP2 & $1(2)$ & $0(0)$ & $0.5^{\mathrm{C}}$ & $2.18(0.15-67)^{d}$ \\
\hline \multicolumn{5}{|l|}{ Combinations of three or more antigens } \\
\hline LDH + Cypin + STIP1 & $5(12)$ & $0.6(1)$ & $0.0371^{c}$ & $3.8(0.96-29.1)^{\mathrm{d}}$ \\
\hline LDH + STIP1 + CRMP1 & $5(13)$ & $0(0)$ & $0.0025^{c}$ & $8.3(1-293)^{d}$ \\
\hline LDH + Cypin + STIP1 + CRMP1 & $2(5)$ & $0(0)$ & $0.16^{\mathrm{c}}$ & $3.1(0.37-124)^{d}$ \\
\hline LDH + Cypin + YBX1 + STIP1 & $2(4)$ & $0(0)$ & $0.16^{\mathrm{c}}$ & $3.1(0.37-124)^{d}$ \\
\hline $\mathrm{LDH}+$ Cypin + YBX $1+$ STIP1 + CRMP2 & $0.4(1)$ & $0(0)$ & $1^{\mathrm{C}}$ & $1.82(0.07-45)^{d}$ \\
\hline $\mathrm{LDH}+\mathrm{YBX} 1+\mathrm{STIP} 1+\mathrm{CRMP} 1+\mathrm{CRMP} 2$ & $0.4(1)$ & $0(0)$ & $1^{\mathrm{c}}$ & $1.82(0.07-45)^{d}$ \\
\hline Cypin + STIP1 + CRMP1 & $7(18)$ & $0.6(1)$ & $0.0025^{\mathrm{c}}$ & $5.8(1.5-43.9)^{d}$ \\
\hline Cypin + YBX1 + STIP1 + CRMP1 & $2(4)$ & $0(0)^{e}$ & $0.16^{\mathrm{c}}$ & $3.1(0.37-124)^{d}$ \\
\hline Cypin + YBX1 + STIP1 + CRMP2 & $1(2)$ & $0(0)$ & $0.5^{\mathrm{c}}$ & $2.18(0.15-67)^{d}$ \\
\hline Cypin + YBX1 + STIP1 + CRMP1 + CRMP2 & $0.4(1)$ & $0(0)$ & $1^{\mathrm{c}}$ & $1.82(0.07-45)^{d}$ \\
\hline YBX1 + STIP1 + CRMP2 & $1(2)$ & $0(0)$ & $0.5^{\mathrm{c}}$ & $2.18(0.15-67)^{d}$ \\
\hline YBX1 + STIP1 + CRMP1 + CRMP2 & $1(2)$ & $0(0)$ & $0.5^{\mathrm{c}}$ & $2.18(0.15-67)^{d}$ \\
\hline All specific combinations combined & $23(56)^{f}$ & $1(2)$ & $<0.0001^{\mathrm{c}}$ & $21.7(5.2-90)$ \\
\hline
\end{tabular}

Abbreviations: ASD, autism spectrum disorder; AU, autism; CI, confidence interval; CRMP, collapsin response mediator protein; LDH, lactate dehydrogenase; OR, odds ratio; STIP1, stress-induced phosphoprotein 1; TD, typically developing; YBX1, Y-box-binding protein.

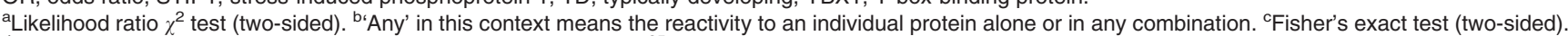
${ }^{\mathrm{d}}$ Small sample size adjustment of OR and $95 \%$ confidence limits, ${ }^{25}$ implemented using R code (http://rss.acs.unt.edu/Rdoc/library/epitools/html/oddsratio.html).

eYBX1 adds specificity to Cypin/STIP1/CRMP1 pattern. ${ }^{\mathrm{f}}$ Takes into account only those mothers with reactivity not represented in more than one pattern. 
Behavioral correlations. To characterize the potential association between maternal antibody status and child behavior, scores on the ABC, Mullen Scales of Early Learning and Vineland Adaptive Behavior Scales were compared between offspring of MAR-positive mothers and those possessing no antibody reactivity; both the ASD and TD subjects were assessed with these instruments. We also analyzed the Autism Diagnostic Observation Schedule and Social Communication Questionnaire behavior scales in the ASD group, but there were no significant associations found with any of the band patterns. In addition, although no significant differences in subscale or composite scores were noted for the Mullen Scales of Early Learning or the Vineland Adaptive Behavioral Scales, increased impairments in the stereotypical behavior subscale of the $A B C$ were observed for children of mothers possessing antibodies reactive to $\mathrm{LDH}$ in any context $(P=0.024)$, CRMP1 (trending toward significance; $P=0.055)$, as well as combined reactivity to LDH and STIP1 $(P=0.015)$, or LDH/STIP1/CRMP1 $(P=0.007)$ in comparison to children with an ASD whose mothers lack IgG reactivity to these antigens. In addition, increased overall impairment was reflected in the composite $A B C$ score for children of mothers reactive against $L D H$ and CRMP1 $(P=0.046)$ as well as LDH/STIP1/CRMP1 (trending toward significance; $P=0.06)$.

\section{Discussion}

Several studies have implicated maternal immune dysregulation during pregnancy in association with ASD. Prominent among them are reports of maternal antibodies that react against fetal brain proteins. ${ }^{6-8,13-16,26-28}$ The hypothesis is that gestational exposure to maternal antibodies directed against proteins abundantly expressed in the fetal brain could lead to alterations in the neurodevelopment characteristic of ASD is further supported by the identification of specific IgG reactivity to LDH, YBX1, cypin, STIP1, CRMP1 and CRMP2, six proteins highly expressed in developing brain. Thus, MAR autoantibodies could represent one mechanism underlying the development of one or more of the ASD core features in a subgroup of cases. Moreover, with their exceptionally high specificity, several of the MAR autoantibody profiles could serve as the first true biomarkers of ASD risk.

Facilitated passage of IgG antibodies during human gestation is a well-characterized phenomenon that provides passive protection for the newborn child, ${ }^{29}$ which persists in the child up to 6 months post-natally. ${ }^{30}$ However, autoantibodies that react to fetal 'self' proteins can also cross the placenta. $^{31}$ In addition, the blood-brain barrier is not fully mature during early brain development, and maternal IgG has been found within mouse ${ }^{32}$ and human fetal brains. ${ }^{7}$ Dalton et $a .^{13}$ first observed that when plasma from a mother of multiple children with ASD possessing reactivity to rodent Purkinje cells was administered to mid-gestation mice, behavioral deficits were present in the offspring. In another study, ${ }^{6}$ mothers and their ASD-affected children were found to have consistent patterns of antibody reactivity against rat prenatal (day 18) brain proteins in contrast to control mothers. We recently employed a passive transfer rodent model using a single gestational exposure to an intravenous dose of purified, brain-reactive IgG antibodies from individual mothers of ASD children or mothers with typically developing children. Growth and behavioral outcomes in offspring revealed alterations in early growth trajectories, significantly impaired motor and sensory development, and increased anxiety. ${ }^{18}$ Thus, there is compelling evidence that maternally derived antibodies could lead to neurodevelopmental alterations in offspring.

Consistent with our early studies on fetal brain homogenates, each of the identified antigens is expressed at significant levels in the human fetal brain ${ }^{33}$ and has an established role in neurodevelopment. For example, cypin is an enzyme with guanine deaminase activity that has an important role in dendritic branching of hippocampal neurons. ${ }^{34}$ It was also recently reported that the postsynaptic protein PSD-95 and cypin regulate dendrite patterning in hippocampal neurons, where cypin promotes microtubule assembly and PSD-95 disrupts microtubule organization. The authors further demonstrated that cypin regulates the density of dendritic spines/filopodia through overexpression/ knockdown experiments. The number of protrusions per dendrite decreased with an overexpression of cypin, whereas a cypin knockdown resulted in an increase in spines/filopodia on the dendrite. ${ }^{35}$ In another study, exposure to brain-derived neurotrophic factor, an important mediator of dendrite arborization and a factor previously implicated in ASD, increases cypin in cultured rat hippocampal neurons. Thus, one can speculate that interfering with cypin through autoantibody exposure during neurodevelopment could affect dendrite formation, potentially increasing the number of dendritic spines in certain regions of the brain. This is consistent with recent data describing spine dysmorphogenesis in ASD where analysis of post-mortem ASD human brain tissue demonstrated an increase in spine density on apical dendrites of pyramidal neurons in a subset of individuals. ${ }^{36}$

STIP1, which is first detected in the developing nervous system, ${ }^{37}$ is a major ligand of the cellular prion protein $(\operatorname{PrP}(\mathrm{C}))$, and together these two proteins mediate neuritogenesis in cultured hippocampal neurons. ${ }^{38}$ Further, protein synthesis in neurons is enhanced via the binding of $\operatorname{PrP}(\mathrm{C})$ with STIP, and the neuroprotection and neuritogenesis mediated by $\operatorname{PrP}(\mathrm{C})$-STI1 engagement are dependent upon this protein increase. ${ }^{39}$ Inhibition of this interaction leads to impaired memory formation in rodents. ${ }^{40} \mathrm{~A}$ recent report also describes the presence of autoantibodies to STIP1 in the blood and CSF of patients with neuro-Behçet's disease, ${ }^{41}$ a chronic recurrent inflammatory disorder with central nervous system involvement that occurs in 5-10\% of Behçet's disease patients. $^{41}$

The group of CRMPs 1-5 are thought to contribute to semaphorin-induced growth cone collapse. ${ }^{42}$ One of the MAR-specific proteins in this group, CRMP1, is highly expressed in the developing brain and is required for proper cell migration and growth cone collapse. ${ }^{42,43}$ It has also been recently demonstrated that CRMP1 and CRMP2 synergistically control dendritic projection, ${ }^{44}$ whereas knockdown of CRMP1 in spinal cord cultures leads to a dramatic reduction in cell survival. ${ }^{45}$ CRMP2 was originally identified as a signaling molecule required for growth cone collapse of dorsal root ganglion neurons in response to a repulsive guidance cue by semaphorin-3A. CRMP2 was also reported to have a positive 
effect on axonal extension and to have a critical role in axondendrite specification and axon regeneration. ${ }^{42}$ Further, blocking experiments suggest that inhibition of CRMP2 does not change the number of neurites, but rather increases the number of cells bearing neurites as well as neurite length. ${ }^{42}$ These findings are of interest when one considers the magnetic resonance imaging studies noting changes in brain growth in children with autism ${ }^{46-48}$ and further studies are currently underway to determine the relationship between MAR autoantibodies and brain growth in ASD. Interestingly, the CRMP1 gene has been associated with the anhedonia endophenotype of schizophrenia in a DISC1 (Disrupted-inschizophrenia 1) gene-dependent manner, and a CRMP1 epitope found in brains from schizophrenia patients is thought to potentially discriminate schizophrenia-derived peripheral blood lymphoblasts from those of normal controls. ${ }^{49}$ CRMP2 has also been associated with several neuropathologic or psychiatric conditions, including Alzheimer's disease and schizophrenia, through genetic polymorphisms, changes in protein expression, post-translational modifications or through protein/protein interactions. ${ }^{50}$

YBX1 is involved in a number of cellular processes, including proliferation, differentiation and the cellular stress response (reviewed in Eliseeva et al. ${ }^{51}$ ). YBX1 functions both in the cytoplasm and in the cell nucleus and it can also be secreted from cells and interact with cell surface receptors to activate intracellular signaling. With respect to fetal brain, YBX1 has an important role in late embryogenesis through in the enhanced cell neuronal motility and migration required for successful neural tube formation. YBX1 mRNA is highly expressed in testicular and fetal brain cells, as well as early precursors of erythroid and lymphoid cells. Interestingly, YBX1 has been found to interact with the Rett Syndrome gene, MeCP2, where it is thought to have RNA splicing activity. YBX1 has also been shown to interact with FMPR, a regulator of $m R N A$ transport and translation, that when absent or defective, gives rise to Fragile-X Syndrome (reviewed in Eliseeva et al. $^{51}$ ).

The enzyme LDH is found in the rodent fetal brain ${ }^{52}$ where it functions in cellular metabolism. Although autoantibodies targeting LDH have not yet been shown to directly have a role in altering neurodevelopment, such autoantibodies have been identified in sera from individuals exposed to the industrial solvent trichloroethylene. ${ }^{53}$ This observation is of interest with respect to the ontogeny of the maternal autoantibodies identified in this report. Circulating LDH levels are often used as a measure of necrotic cell damage following toxic exposures $^{54}$ and viral infections, ${ }^{55}$ and in this inflammatory context could give rise to an antibody-producing immune response in the mother. For example, a mouse model of maternal immune activation where maternal inflammation during gestation results in behavioral changes in the offspring, demonstrated an increase in LDH-B in the prefrontal cortex. ${ }^{56}$ The relatively high prevalence $(28 \%$ overall among mothers of children with ASD) indicates a critical need to define the role for LDH in the developing brain.

Our finding of an increased incidence of stereotypic behavior in children whose mothers have autoantibodies targeting LDH, CRMP1 and STIP1 suggests a role for these proteins in a core behavioral feature of ASD. Interestingly, this

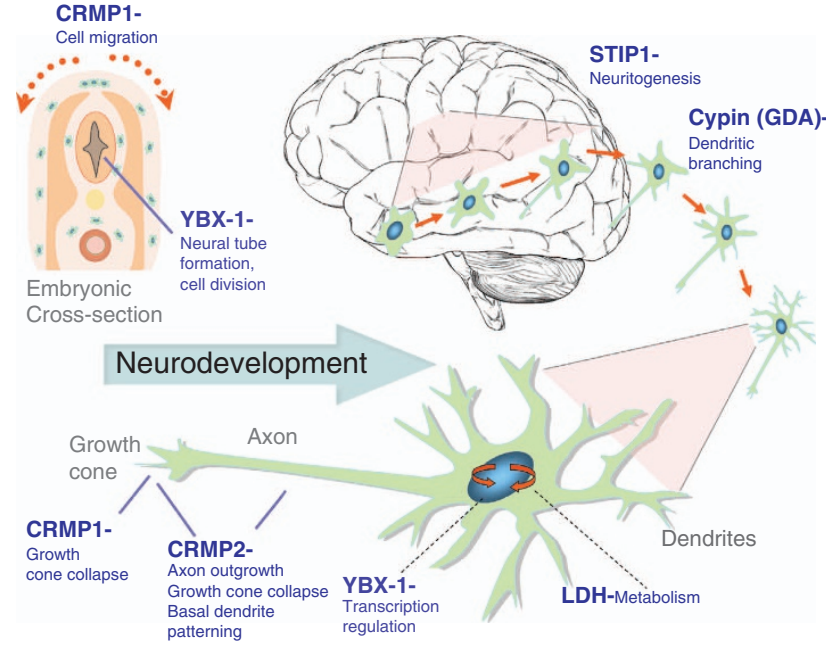

Figure 4 Schematic model depicting the neurodevelopmental roles for each of the maternal autoantibody target proteins. Disruption of protein function or quantity may negatively alter neurodevelopment, potentially leading to the core features associated with autism. There is likely an additive effect as illustrated by the autism spectrum disorder specificity of the various autoantibody combinations. CRMP, collapsin response mediator protein; LDH, lactate dehydrogenase; STIP1, stressinduced phosphoprotein 1; YBX1, Y-box-binding protein.

result is consistent with behavior observations from our earlier non-human primate gestational IgG passive transfer study ${ }^{16}$ using some of the same antibody sources as those discussed herein. The stereotypic behavior in Rhesus monkey offspring noted after gestational exposure, coupled with the increased stereotypical behavior among children of mothers analyzed in this study, suggests that a subset of MAR autoantibodies may be pathologically significant, eliciting changes in all aspects of $A S D$, but with a particularly strong effect in the behavioral domain. Other behavioral differences have been characterized between ASD cases based on maternal autoantibody status, such as increased irritability and impaired use of expressive language ${ }^{8}$ using a similar study population. The inconsistencies between our two studies regarding behaviorautoantibody associations may stem from the increased detection sensitivity afforded by the use of pure antigens rather than the crude fetal brain protein preparations employed in previous studies. Another possibility is the presence of additional autoantigens that we have not yet discovered, and that could be related to impaired use of expressive language. We are currently exploring these additional candidates.

Each of the target autoantigens defined herein is known to have a critical role in the developing brain and interference with the level or function of more than one of them could act synergistically to change the trajectory of brain development. Figure 4 illustrates the known functions for each of the antigenic proteins during neuron migration and development. The effect of MAR autoantibodies could occur through a direct antigen-antibody interaction, thereby either decreasing the abundance of or causing functional interference of the target proteins. Alternatively, the presence of these maternal antibodies may merely serve as a biomarker of cell destruction. One of the limitations of the current study is that all of the clinical samples used for analysis of reactivity to the 
target autoantigens were obtained from mothers of children at the time of the child's diagnosis (retrospective sample). Studies are currently underway to assess the predictive value of the MAR autoantibodies in a prospective study sample. Addressing the basis for the association between maternal autoantibodies and autism risk in the child is also of urgent concern. Animal studies involving targeted autoantibody exposure throughout gestation are currently underway to address the hypothesis that pre-natal exposure to these specific antibodies leads to behavioral alterations in offspring. The clinical significance of these findings may be twofold: (1) early diagnosis of a MAR-ASD child would allow for early behavioral intervention and (2) if pathologically significant, medical interventions that would limit fetal exposure to these antibodies might prove helpful in reducing risk of ASD symptom development in the children of affected mothers.

\section{Conflict of interest}

The corresponding (Van de Water) and first (Braunschweig) authors have a published patent on the proteins described in this manuscript. Van de Water is a consultant for Pediatric Bioscience, a company that has licensed this technology from UC Davis. The remaining authors declare no conflict of interest.

Acknowledgements. This work was supported by NIEHS 1 P01 ES11269-01, the US Environmental Protection Agency (USEPA) through the Science to Achieve Results (STAR) program (Grant R829388), NIEHS 1 R01ES015359, the UC Davis M.I.N.D. Institute and Autism Speaks graduate fellowship (DB) and US patent 8,383,360 B2. We thank Elizabeth Fox for her help in reading and editing this manuscript.

1. Investigators CAaDDMNSYP. Prevalence of autism spectrum disorders-autism and developmental disabilities monitoring network, 14 Sites, United States, 2008. MMWR Surveill Summ 2012; 61: 1-19.

2. APA Diagnostic and Statistical Manual of Mental Disorders: DSM-IV Text Revision. American Psychiatric Association: Washington, DC.

3. Goines P, Van de Water J. The immune system's role in the biology of autism. Curr Opin Neurol 2010; 23: 111-117.

4. Heuer L, Ashwood P, Schauer J, Goines P, Krakowiak P, Hertz-Picciotto I et al. Reduced levels of immunoglobulin in children with autism correlates with behavioral symptoms. Autism Res 2008; 1: 275-283.

5. Vargas DL, Nascimbene C, Krishnan C, Zimmerman AW, Pardo CA. Neuroglial activation and neuroinflammation in the brain of patients with autism. Ann Neurol 2005; 57: 67-81.

6. Zimmerman AW, Connors SL, Matteson KJ, Lee LC, Singer HS, Castaneda JA et al. Maternal antibrain antibodies in autism. Brain Behav Immun 2007; 21: 351-357.

7. Braunschweig D, Ashwood P, Krakowiak P, Hertz-Picciotto I, Hansen R, Croen LA et al. Autism: maternally derived antibodies specific for fetal brain proteins. Neurotoxicology 2008; 29: 226-231.

8. Braunschweig D, Duncanson P, Boyce R, Hansen R, Ashwood P, Pessah IN et al. Behavioral correlates of maternal antibody status among children with autism. $J$ Autism Dev Disord 2011; 42: 1435-1445.

9. Heuer L, Braunschweig D, Ashwood P, Van de Water J, Campbell DB. Association of a MET genetic variant with autism-associated maternal autoantibodies to fetal brain proteins and cytokine expression. Translational Psychiatry 2011; 1: e48.

10. Simister NE. Placental transport of immunoglobulin G. Vaccine 2003; 21: 3365-3369.

11. Malek A, Sager R, Kuhn $P$, Nicolaides KH, Schneider $H$. Evolution of maternofetal transport of immunoglobulins during human pregnancy. Am J Reprod Immunol 1996; 36: 248-255.

12. Bake S, Friedman JA, Sohrabji F. Reproductive age-related changes in the blood brain barrier: expression of IgG and tight junction proteins. Microvasc Res 2009; 78: 413-424.

13. Dalton P, Deacon R, Blamire A, Pike M, McKinlay I, Stein J et al. Maternal neuronal antibodies associated with autism and a language disorder. Ann Neurol 2003; 53: 533-537.

14. Singer HS, Morris C, Gause C, Pollard M, Zimmerman AW, Pletnikov M. Prenatal exposure to antibodies from mothers of children with autism produces neurobehavioral alterations: a pregnant dam mouse model. J Neuroimmunol 2009; 211: 39-48.
15. Braunschweig D, Golub MS, Koenig CM, Qi L, Pessah IN, Van de Water J et al. Maternal autism-associated IgG antibodies delay development and produce anxiety in a mouse gestational transfer model. J Neuroimmunol 2012; 252: 56-65.

16. Martin LA, Ashwood P, Braunschweig D, Cabanlit M, Van de Water J, Amaral DG. Stereotypies and hyperactivity in rhesus monkeys exposed to $\mathrm{lgG}$ from mothers of children with autism. Brain Behav Immun 2008; 22: 806-816.

17. Hertz-Picciotto I, Croen LA, Hansen R, Jones CR, van de Water J, Pessah IN. The CHARGE study: an epidemiologic investigation of genetic and environmental factors contributing to autism. Environ Health Perspect 2006; 114: 1119-1125.

18. Braunschweig D, Duncanson P, Boyce R, Hansen R, Ashwood P, Pessah IN et al. Behavioral correlates of maternal antibody status among children with autism. $J$ Autism Dev Disord 2012; 42: 1435-1445.

19. Lord C, Rutter M, diLavore P, Risi S. Autism Diagnostic Observation Schedule Manual. Western Psychological Services: Los Angeles, CA, 2003.

20. Le Couteur A, Lord C, Rutter M. The Autism Diagnostic Interview, Revised (ADI-R). Western Psychological Services: Los Angeles, CA, 1989.

21. Mullen E. Mullen Scale of Early Learning. American Guidance Service: Circle Pines, MN, 1995.

22. Sparrow SB, Cicchetti DA, Vineland DV. Adaptive Behavior Scales Survey Form Manual. American Guidance Service: Circle Pines, MN, 1985.

23. Aman M, Singh N. Abberant Behavioral Checklist-Community Supplementary Manual Slosson Educational Publications: East Aurora, NY, 1986.

24. Subbiah M, Srinivasan MR. Classification of $2 \times 2$ sparse data sets with zero cells. Statist Probab Lett 2008; 78: 3212-3215.

25. Jewell NP, Kalbfleisch JD. Maximum likelihood estimation of ordered multinomial parameters. Biostatistics 2004; 5: 291-306.

26. Croen LA, Braunschweig D, Haapanen L, Yoshida CK, Fireman B, Grether JK et al. Maternal mid-pregnancy autoantibodies to fetal brain protein: the early markers for autism study. Biol Psychiatry 2008; 64: 583-588.

27. Singer HS, Morris CM, Gause CD, Gillin PK, Crawford S, Zimmerman AW. Antibodies against fetal brain in sera of mothers with autistic children. $J$ Neuroimmunol 2008; 194: $165-172$.

28. Warren RP, Cole P, Odell JD, Pingree CB, Warren WL, White E et al. Detection of maternal antibodies in infantile autism. J Am Acad Child Adolesc Psychiatry 1990; 29: 873-877.

29. Garty BZ, Ludomirsky A, Danon YL, Peter JB, Douglas SD. Placental transfer of immunoglobulin G subclasses. Clin Diagn Lab Immunol 1994; 1: 667-669.

30. Heininger U, Desgrandchamps D, Schaad UB. Seroprevalence of Varicella-Zoster virus IgG antibodies in Swiss children during the first 16 months of age. Vaccine 2006; 24: 3258-3260.

31. Tincani A, Rebaioli CB, Taglietti M, Shoenfeld Y. Heart involvement in systemic lupus erythematosus, anti-phospholipid syndrome and neonatal lupus. Rheumatology (Oxford) 2006; 45(Suppl 4): iv8-13.

32. Lee JY, Huerta PT, Zhang J, Kowal C, Bertini E, Volpe BT et al. Neurotoxic autoantibodies mediate congenital cortical impairment of offspring in maternal lupus. Nat Med 2009; 15: 91-96.

33. Wheeler DL, Church DM, Federhen S, Lash AE, Madden TL, Pontius JU et al. Database resources of the National Center for Biotechnology. Nucleic Acids Res 2003; 31: $28-33$.

34. Akum BF, Chen M, Gunderson SI, Riefler GM, Scerri-Hansen MM, Firestein BL. Cypin regulates dendrite patterning in hippocampal neurons by promoting microtubule assembly. Nat Neurosci 2004; 7: 145-152.

35. Tseng CY, Firestein BL. The role of PSD-95 and cypin in morphological changes in dendrites following sublethal NMDA exposure. J Neurosci 2011; 31: 15468-15480.

36. Hutsler JJ, Zhang $\mathrm{H}$. Increased dendritic spine densities on cortical projection neurons in autism spectrum disorders. Brain Res 2010; 1309: 83-94.

37. Hajj GN, Santos TG, Cook ZS, Martins VR. Developmental expression of prion protein and its ligands stress-inducible protein 1 and vitronectin. J Comp Neurol 2009; 517: 371-384.

38. Lopes MH, Hajj GN, Muras AG, Mancini GL, Castro RM, Ribeiro KC et al. Interaction of cellular prion and stress-inducible protein 1 promotes neuritogenesis and neuroprotection by distinct signaling pathways. J Neurosci 2005; 25: 11330-11339.

39. Roffé M, Beraldo FH, Bester R, Nunziante M, Bach C, Mancini G et al. Prion protein interaction with stress-inducible protein 1 enhances neuronal protein synthesis via mTOR. Proc Natl Acad Sci USA 2010; 107: 13147-13152.

40. Coitinho AS, Lopes MH, Hajj GN, Rossato JI, Freitas AR, Castro CC et al. Short-term memory formation and long-term memory consolidation are enhanced by cellular prion association to stress-inducible protein 1. Neurobiol Dis 2007; 26: 282-290.

41. Vural $B$, Uğurel E, Tüzün E, Kürtüncü M, Zuliani L, Cavuş F et al. Anti-neuronal and stressinduced-phosphoprotein 1 antibodies in neuro-Behçet's disease. J Neuroimmunol 2011; 239: 91-97.

42. Quach TT, Duchemin AM, Rogemond V, Aguera M, Honnorat J, Belin MF et al. Involvement of collapsin response mediator proteins in the neurite extension induced by neurotrophins in dorsal root ganglion neurons. Mol Cell Neurosci 2004; 25: 433-443.

43. Charrier E, Reibel S, Rogemond V, Aguera M, Thomasset N, Honnorat J. Collapsin response mediator proteins (CRMPs): involvement in nervous system development and adult neurodegenerative disorders. Mol Neurobiol 2003; 28: 51-64. 
44. Yamashita N, Ohshima T, Nakamura F, Kolattukudy P, Honnorat J, Mikoshiba K et al. Phosphorylation of CRMP2 (collapsin response mediator protein 2) is involved in prope dendritic field organization. J Neurosci 2012; 32: 1360-1365.

45. Kurnellas MP, Li H, Jain MR, Giraud SN, Nicot AB, Ratnayake A et al. Reduced expression of plasma membrane calcium ATPase 2 and collapsin response mediator protein promotes death of spinal cord neurons. Cell Death Differ 2010; 17: 1501-1510.

46. Nordahl CW, Lange N, Li DD, Barnett LA, Lee A, Buonocore MH et al. Brain enlargement is associated with regression in preschool-age boys with autism spectrum disorders. Proc Natl Acad Sci USA 2011; 108: 20195-20200.

47. Nordahl CW, Scholz R, Yang X, Buonocore MH, Simon T, Rogers S et al. Increased rate of amygdala growth in children aged 2 to 4 years with autism spectrum disorders: a longitudinal study. Arch Gen Psychiatry 2012; 69: 53-61.

48. Aoki Y, Kasai K, Yamasue H. Age-related change in brain metabolite abnormalities in autism: a meta-analysis of proton magnetic resonance spectroscopy studies. Trans/ Psychiatry 2012; 2: e69.

49. Bader V, Tomppo L, Trossbach SV, Bradshaw NJ, Prikulis I, Leliveld SR et al. Proteomic genomic and translational approaches identify CRMP1 for a role in schizophrenia and its underlying traits. Hum Mol Genet 2012; 21: 4406-4418.

50. Hensley K, Venkova K, Christov A, Gunning W, Park J. Collapsin response mediator protein-2: an emerging pathologic feature and therapeutic target for neurodisease indications. Mol Neurobiol 2011; 43: 180-191.

51. Eliseeva IA, Kim ER, Guryanov SG, Ovchinnikov LP, Lyabin DN. Y-box-binding protein 1 (YB-1) and its functions. Biochemistry (Mosc) 2011; 76: 1402-1433.
52. Hashimoto T, Hussien R, Cho HS, Kaufer D, Brooks GA. Evidence for the mitochondrial lactate oxidation complex in rat neurons: demonstration of an essential component of brain lactate shuttles. PLoS One 2008; 3: e2915.

53. Liu J, Xing $\mathrm{X}$, Huang $\mathrm{H}$, Jiang $\mathrm{Y}, \mathrm{He} \mathrm{H}, \mathrm{Xu} X$ et al. Identification of antigenic proteins associated with trichloroethylene-induced autoimmune disease by serological proteome analysis. Toxicol Appl Pharmacol 2009; 240: 393-400.

54. Silva RF, Falcao AS, Fernandes A, Gordo AC, Brito MA, Brites D. Dissociated primary nerve cell cultures as models for assessment of neurotoxicity. Toxicol Lett 2006; 163: $1-9$.

55. Shin SY, Kim JH, Kim HS, Kang YA, Lee HG, Kim JS et al. Clinical characteristics of Korean pediatric patients critically ill with influenza A (H1N1) virus. Pediatr Pulmonol 2010; 45: 1014-1020.

56. Deng MY, Lam S, Meyer U, Feldon J, Li Q, Wei R et al. Frontal-subcortical protein expression following prenatal exposure to maternal inflammation. PLoS One 2011; 6 : e16638.

(c) (i) $(\Theta)$ Translational Psychiatry is an open-access journal By ${ }_{\text {nc }}$ po published by Nature Publishing Group. This work is licensed under a Creative Commons Attribution-NonCommercialNoDerivs 3.0 Unported License. To view a copy of this license, visit http://creativecommons.org/licenses/by-nc-nd/3.0/ 